\title{
Characterization of Inclusions in Evolution of Sodium Sulfate Using Terahertz Time-domain Spectroscopy
}

\author{
Rima BAO, ${ }^{* \dagger}$ Zhikui WU,* Hao LI,** Fang WANG,* Xinyang MiAo,* and Chengjing FenG* \\ *Beijing Key Laboratory of Optical Detection Technology for Oil and Gas, China University of Petroleum, \\ Beijing 102249, P. R. China \\ **Petroleum Exploration \& Production Research Institute, China Petroleum \& Chemical Corporation (SINOPEC), \\ Beijing 100083, P. R. China
}

\begin{abstract}
The study of fluid inclusion is one of the important means to understanding the evolution of mineral crystals, and can therefore provide original information of mineral evolution. In the process of evolution, outside factors such as temperature and pressure, directly affect the number and size of inclusions, and thus are related to the properties of crystals. In this paper, terahertz time-domain spectroscopy (THz-TDS) was used to detect sodium sulfate crystals with different growth temperatures, and absorption coefficient spectra of the samples were obtained. It is suggested that the evolution of sodium sulfate could be divided into two stages, and $80^{\circ} \mathrm{C}$ was the turning point. X-ray diffraction (XRD) and polarizing microscopy were used to support this conclusion. The research showed that THz-TDS could characterize the evolution of mineral crystals, and it had a unique advantage in terms of crystal evolution.
\end{abstract}

Keywords Inclusions, crystal growth, sodium sulfate, THz-TDS

(Received March 22, 2017; Accepted June 5, 2017; Published September 10, 2017)

\section{Introduction}

Making an important contribution to the study of the evolution of mineral crystals, research on and applications of fluid inclusions have progressed tremendously over the last three decades. ${ }^{1,2}$ Fluid inclusions are small volumes of ancient fluid trapped in minerals that provide indispensable information about geological processes, from high temperatures at depth towards low temperatures near the Earth's surface. ${ }^{3,4}$ The formation of inclusions is closely related to the mineral crystallization process, which exists throughout all geological processes. Fluid inclusions reveal geologically important information such as temperature, pressure, salinity, density and depth of trapping; and thereby provide direct information about the conditions at which given minerals and rocks are formed. So the study of the inclusions has great significance for clarifying the evolution of crystals.

The study of fluid inclusions has come a long way since their initial description by Sorby (1857). In recent decades, the research of inclusions has made great progress, and many methods have been applied to the study of inclusions, such as homogenization method, burst method and quenching method for measuring temperature; ${ }^{5}$ freezing method for the determination of salinity; laser Raman probes, chromatography, mass spectrometry, ultraviolet fluorescence spectroscopy for the determination of gas and liquid phase composition, ${ }^{6-8}$ and so on. These methods play an important role in the study of inclusions, and promote the development of research on inclusions.

$\dagger$ To whom correspondence should be addressed.

E-mail: brm22@163.com
However, these methods have their shortcomings, for example, invasion to samples, poor accuracy, poor reproducibility. Looking for new methods to study the inclusions is still important.

As a recently arisen spectroscopic method, terahertz timedomain spectroscopy (THz-TDS) uses terahertz waves that range from 0.1 to $10 \mathrm{THz}$, and bridges the gap between infrared and microwave as a light source. ${ }^{9}$ Terahertz waves can penetrate many nonpolar materials, but they do not damage the sample because of the lower photon energy. ${ }^{10}$ In addition, the waves can induce low frequency bond vibrations, crystal phonon vibration, hydrogen-bonding stretches, and torsion vibrations in materials. ${ }^{11}$ In recent decades, because of these unique properties, THz-TDS has attracted more attention and has been employed in various fields, such as biochemistry, safety, medicine quality control, food, petroleum and geological evolution. ${ }^{12-16}$

In this paper, THz-TDS was applied to measure sodium sulfate crystals with different growth temperatures. The change of fluid inclusion and its influence on the evolution of sodium sulfate were characterized by terahertz optical parameters, which was verified by XRD and polarizing microscopy. This paper indicated that THz-TDS could be used to characterize the fluid inclusions in the crystal growth process and provided a new method to characterize crystal evolution.

\section{Experimental}

Crystals of $\mathrm{Na}_{2} \mathrm{SO}_{4}$ were grown by evaporation crystallization. First, a saturated solution of $\mathrm{Na}_{2} \mathrm{SO}_{4}$ was prepared at room temperature. After filtering, it was placed in the drying oven 


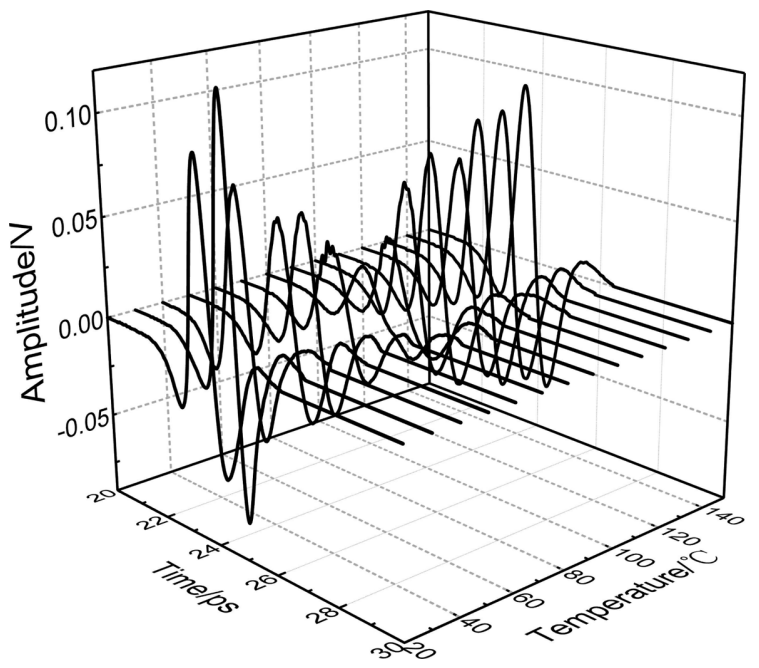

Fig. 1 Terahertz three-dimensional time-domain spectrum of samples.

that had been set to a temperature. Waiting for the solution to dry, sodium sulfate crystals were obtained. In order to get different samples, evaporation temperature was set at 20 to $150^{\circ} \mathrm{C}$ at temperature intervals of $10^{\circ} \mathrm{C}$. In order to eliminate the effect of the particle size, obtained crystals were ground and shifted, which made particle size within $120 \mu \mathrm{m}$. Secondly, samples were made by tabletting with $25 \mathrm{MPa}$ pressure for 2 min. This operation did not destroy the structure of the crystal so that it had no effect on the inclusions. Each sample weighed $2 \mathrm{~g}$, and had an average thickness of $1.352 \mathrm{~mm}$. Finally, the samples were detected by THz-TDS.

In THz-TDS system, terahertz wave was produced by a femtosecond laser triggered photoconductive antenna. Femtosecond laser with center wavelength of $800 \mathrm{~nm}$, repetition frequency of $80 \mathrm{MHz}$, and pulse width of $100 \mathrm{fs}$ was produced by titanium sapphire femtosecond mode-locked pulse laser. Laser pulse could be divided into two beams by a beam splitter, a beam as a pump, to motivate GaAs photoconductive antenna generate $\mathrm{THz}$ pulse, and the other as a probe light, to measure $\mathrm{THz}$ signal by detector. Finally, $\mathrm{THz}$ signal was gathered by phase-locked amplifier controlled by the chopper, and transmitted to the computer for processing. In order to reduce the absorption of water vapor in the air to the $\mathrm{THz}$ wave, dry air was used in the terahertz optical path, with humidity of less $1 \%$ and temperature of $298 \mathrm{~K}$. The dry air in the system was measured by THz-TDS, and the results were taken as reference spectra. The absorption coefficient spectra were calculated automatically according to the samples and the reference spectra by the system.

\section{Results and Discussion}

Growth conditions, such as temperature, pressure, humidity and so on, play a critical role for crystal growth, and are a major cause of different results in crystal growth. This experiment mainly studied the effect of temperature on crystal growth in the temperature range of $20-150^{\circ} \mathrm{C}$. Sodium sulfate crystals obtained by evaporating saturated solution at different temperatures were measured by the THz-TDS system to obtain the terahertz time-domain spectra of samples. Figure 1 shows the three-dimensional time-domain waveforms of samples.

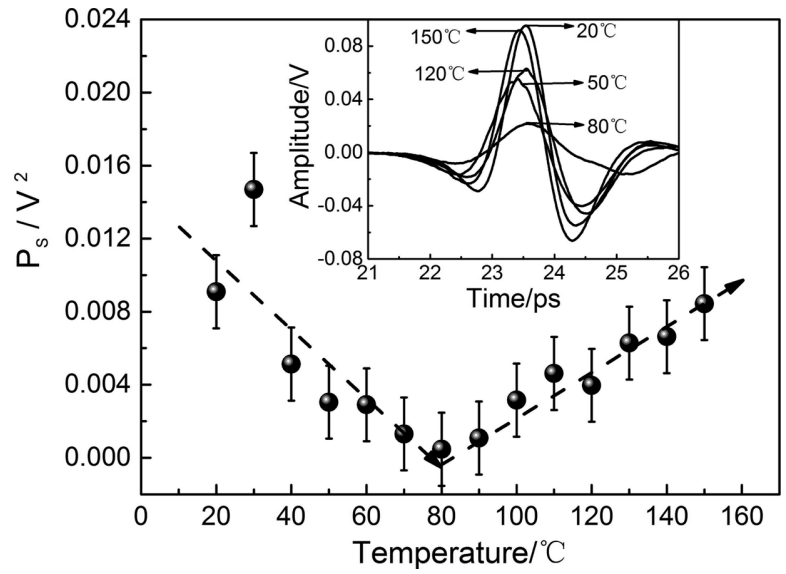

Fig. 2 The relationship curve between the square of the THz e-field $\left(P_{\mathrm{S}}\right)$ and growth temperatures.

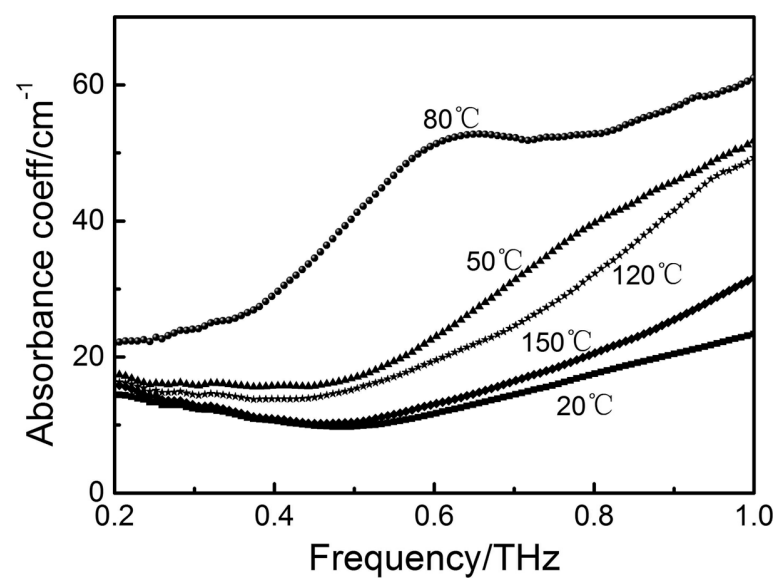

Fig. 3 Absorbance coefficients of samples grown in temperatures of $20,50,80,120$ and $150^{\circ} \mathrm{C}$.

As shown in Fig. 1, there are different terahertz time-domain spectra of sodium sulfate crystals with different temperatures, especially the curve peak. The change of the peak value can be clearly seen from the figure. In order to show the phenomenon more clearly, we plotted a graph between square of the $\mathrm{THz}$ e-field $\left(P_{\mathrm{S}}\right)$ and growth temperatures, as shown in Fig. 2. When the temperature rises from 20 to $80^{\circ} \mathrm{C}, P_{\mathrm{S}}$ decreases linearly from 0.0091 to $0.0004 \mathrm{~V}^{2}$. However, the change of $P_{\mathrm{S}}$ is various when the temperature continues to rise. As the temperature changes from 80 to $150^{\circ} \mathrm{C}, P_{\mathrm{S}}$ linearly increases from 0.0004 to $0.0084 \mathrm{~V}^{2}$. The change can be divided into two parts, and $80^{\circ} \mathrm{C}$ is noted as a turning point.

The energy loss of the $\mathrm{THz}$ waves has a one-to-one correspondence with the absorption coefficient. Figure 3 shows a sample's absorption coefficient spectra within $0.2-1.0 \mathrm{THz}$. There are absorption coefficient curves at growth temperatures of $20,50,80,120$ and $150^{\circ} \mathrm{C}$, respectively. The absorption coefficients increase with the increase of frequency. In addition, samples with different growth temperatures have disparate absorption coefficients. When the growth temperature is increased from 20 to $80^{\circ} \mathrm{C}$, the absorption coefficients increase with grown a rise in temperature. On the contrary, absorption coefficients begin to decline when the growth temperature is 
greater than $80^{\circ} \mathrm{C}$.

To exhibit more clearly, Fig. 4 shows discontinuous absorption coefficients at $0.4,0.5,0.6,0.7$ and $0.8 \mathrm{THz}$, is used to indicate these objective law. At the same growth temperature, the higher the frequency, the greater the absorption coefficients, which can be seen in Fig. 4. Within the temperature range of $20-80^{\circ} \mathrm{C}$, absorption coefficients increase when the growth temperature increases. But when the temperature is greater than $80^{\circ} \mathrm{C}$, it is just the opposite, absorption coefficients decrease with the increase of temperature, which is anastomotic with the $P_{\mathrm{s}}$. However, there is a special case that the absorption coefficient has reached the maximum at $70^{\circ} \mathrm{C}$ rather than at $80^{\circ} \mathrm{C}$ at $0.8 \mathrm{THz}$. The reason for this phenomenon may be the error, and it also may be because the temperature is not divided carefully enough, leading to deviation of the extremum.

In Fig. 5, the X-ray diffractometer is used in order to explore the causes of this phenomenon, whose scanned range is from 5 to 90 degrees. Five samples whose growth temperatures are respectively $20,50,80,120$ and $150^{\circ} \mathrm{C}$ are selected to draw XRD profiles, and XRD curves are positioned perpendicularly

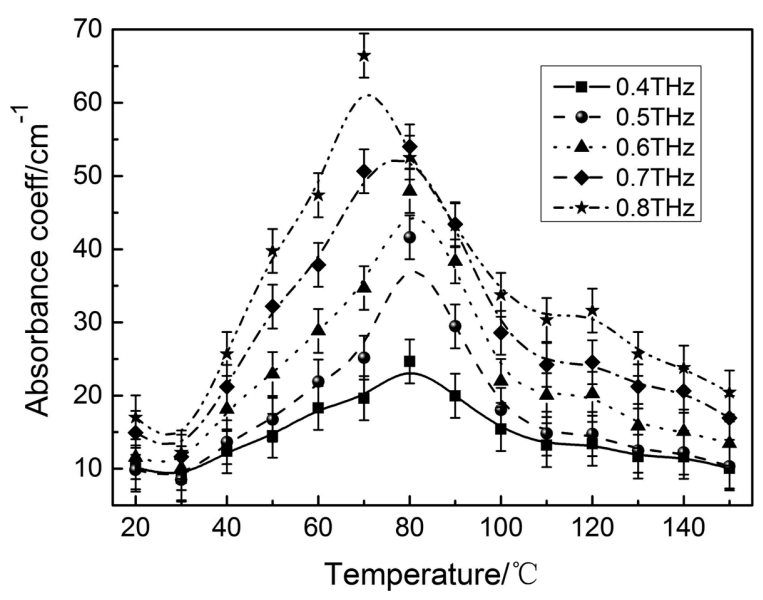

Fig. 4 Absorbance coefficients of samples with different growth temperature in $0.4,0.5,0.6,0.7$ and $0.8 \mathrm{THz}$. in order to show a clear comparison. The result is that XRD peaks of samples are identical to the characteristic peaks of pure sodium sulfate, which shows that there is no crystal water inside sodium sulfate crystals. In other words, samples are $\mathrm{Na}_{2} \mathrm{SO}_{4}$ instead of $\mathrm{Na}_{2} \mathrm{SO}_{4} \cdot 10 \mathrm{H}_{2} \mathrm{O}$ and $\mathrm{Na}_{2} \mathrm{SO}_{4} \cdot 7 \mathrm{H}_{2} \mathrm{O}$, and the above phenomenon is not caused by crystal water.

The cause of this phenomenon may be fluid inclusions inside crystals. Microscope photos of inclusions inside sodium sulfate crystals are as shown in Fig. 6. Most of inclusions are gasliquid inclusions. The number and size of them are disparate because of the different growth temperatures, but the size of them is within $50 \mu \mathrm{m}$. The number of inclusions is less when the growth temperature is below $80^{\circ} \mathrm{C}$, but when the growth temperature is higher than $80^{\circ} \mathrm{C}$, it becomes more and more. This is due to growth temperature. As growth temperature increases, the crystallization rate becomes faster, and the possibility of crystal defect increases. So the number of inclusions becomes more and more. According to microscope photos, the number of inclusions' pixels is divided by the total number of pixels to get the proportion of the inclusions' area. It was measured in order to show more accurately differences of inclusions, and the result is shown in the following Table 1.

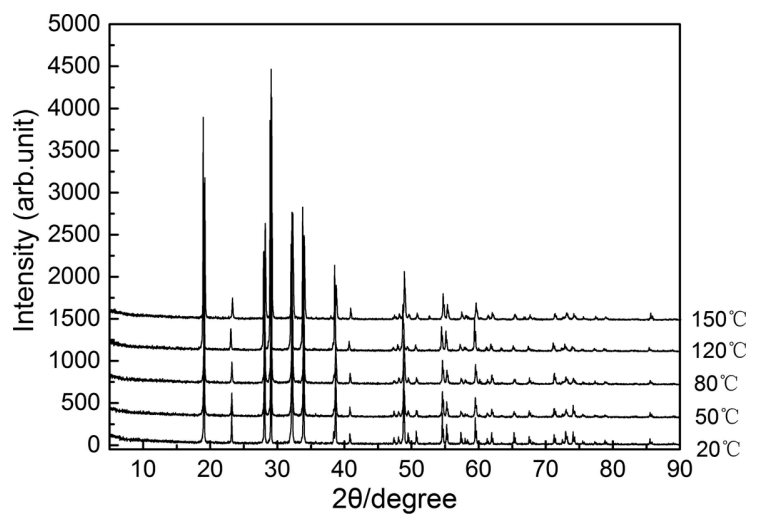

Fig. 5 X-ray diffraction patterns of samples with different growth temperatures.

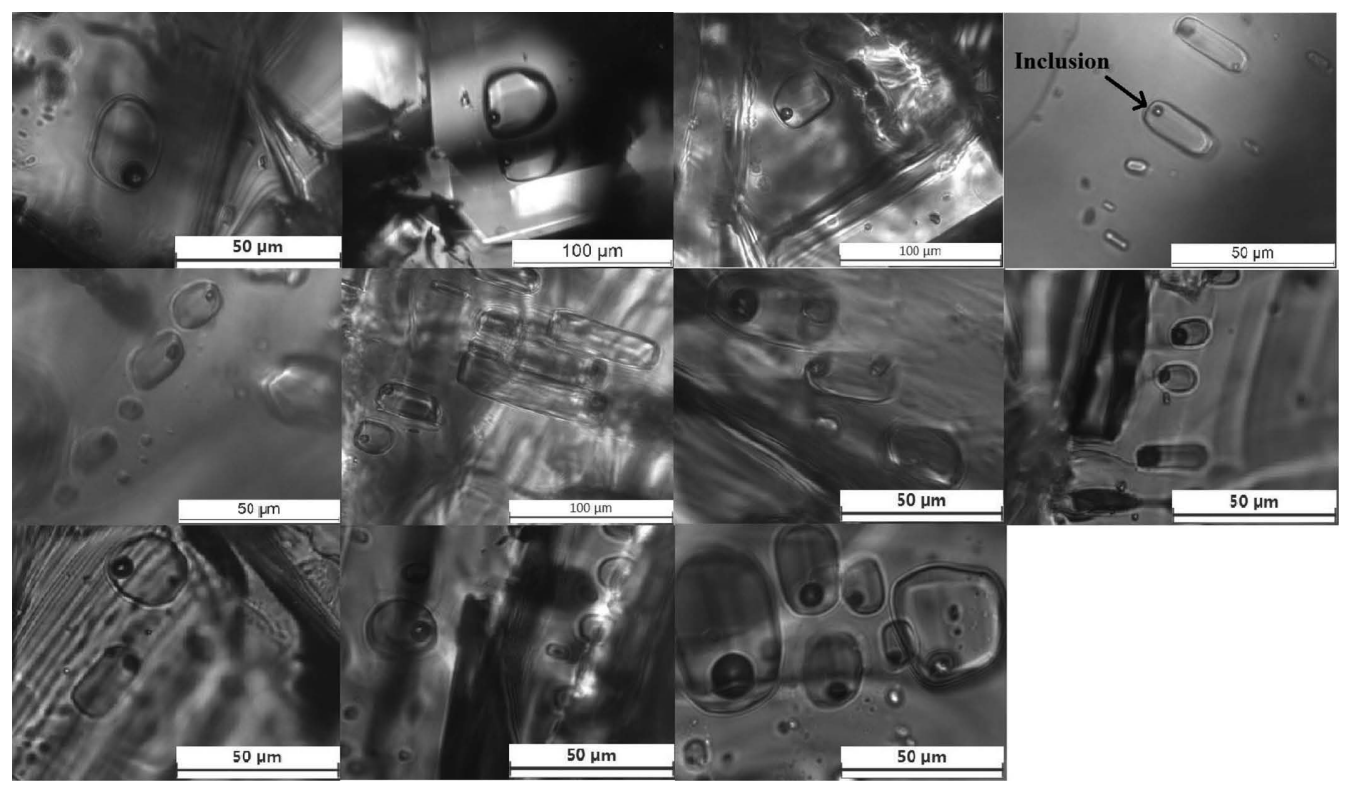

Fig. 6 Microscope photos of fluid inclusions inside samples. 
Table 1 The proportion of inclusions' areas inside samples

\begin{tabular}{cc}
\hline Growth temperature $/{ }^{\circ} \mathrm{C}$ & The proportion of inclusions' areas, \% \\
\hline 40 & 0.355 \\
50 & 0.582 \\
60 & 0.281 \\
70 & 1.286 \\
80 & 1.970 \\
90 & 2.671 \\
110 & 3.449 \\
120 & 4.490 \\
130 & 1.532 \\
140 & 2.555 \\
\end{tabular}

Table 2 Weights of samples before and after drying for $24 \mathrm{~h}$ in $300^{\circ} \mathrm{C}$

\begin{tabular}{cccc}
\hline $\begin{array}{c}\text { Growth } \pm \\
\text { temperature/ } \\
{ }^{\circ} \mathrm{C}\end{array}$ & Before/g & After/g & Change/g \\
\hline 20 & $1.0112 \pm 0.0001$ & $1.0109 \pm 0.0001$ & $0.0003 \pm 0.0002$ \\
30 & $1.0016 \pm 0.0001$ & $1.0011 \pm 0.0001$ & $0.0005 \pm 0.0002$ \\
40 & $1.0139 \pm 0.0001$ & $1.0112 \pm 0.0001$ & $0.0027 \pm 0.0002$ \\
50 & $1.0041 \pm 0.0001$ & $1.0018 \pm 0.0001$ & $0.0023 \pm 0.0002$ \\
60 & $1.0262 \pm 0.0001$ & $1.0236 \pm 0.0001$ & $0.0026 \pm 0.0002$ \\
70 & $1.0121 \pm 0.0001$ & $1.0098 \pm 0.0001$ & $0.0023 \pm 0.0002$ \\
80 & $1.0116 \pm 0.0001$ & $1.0101 \pm 0.0001$ & $0.0015 \pm 0.0002$ \\
90 & $1.0204 \pm 0.0001$ & $1.0190 \pm 0.0001$ & $0.0014 \pm 0.0002$ \\
100 & $1.0096 \pm 0.0001$ & $1.0083 \pm 0.0001$ & $0.0013 \pm 0.0002$ \\
110 & $1.0195 \pm 0.0001$ & $1.0182 \pm 0.0001$ & $0.0013 \pm 0.0002$ \\
120 & $0.9944 \pm 0.0001$ & $0.9929 \pm 0.0001$ & $0.0015 \pm 0.0002$ \\
130 & $0.9837 \pm 0.0001$ & $0.9821 \pm 0.0001$ & $0.0016 \pm 0.0002$ \\
140 & $1.0144 \pm 0.0001$ & $1.0124 \pm 0.0001$ & $0.0020 \pm 0.0002$ \\
150 & $0.9884 \pm 0.0001$ & $0.9861 \pm 0.0001$ & $0.0023 \pm 0.0002$ \\
\hline
\end{tabular}

It can be seen that proportions of inclusions' area are mainly increased with the increase of growth temperature. When the growth temperature is below $80^{\circ} \mathrm{C}$, the proportions of inclusions' area are minor, but when the growth temperature is higher than $80^{\circ} \mathrm{C}$, they become more. So the growth temperature of the crystal has a great influence on inclusions.

Fluid inclusions contain water, and water has a strong absorption for terahertz waves. In order to measure the quantity of water in inclusions, $1 \mathrm{~g}$ was extracted from every sample and dried for $24 \mathrm{~h}$ in the drying oven whose temperature was set at $300^{\circ} \mathrm{C}$, which ensured that inclusions were completely broken and water inside them was completely evaporated. Electronic balance was used to weigh the samples after drying, and the results are shown in Table 2 . The quantity of water inside inclusions increased with the rise of growth temperature when the growth temperature was below $80^{\circ} \mathrm{C}$. But at temperatures higher than $80^{\circ} \mathrm{C}$, the quantify of water basically remained unchanged, which also indicated the content of the gas inside inclusions grew.

In summary, the number and size of inclusions were small when the growth temperature was below $80^{\circ} \mathrm{C}$, and the quantity of water inside inclusions increased with the rise of growth temperature. So absorption coefficients of samples had an increasing trend because of the absorption of water for terahertz. On the contrary, with growth temperatures above $80^{\circ} \mathrm{C}$, the number of inclusions inside samples multiplied, and the proportions of inclusions' area were greater. But the quantity of water inside inclusions basically remained unchanged, which indicated the content of the gas grew with the increase of growth temperatures. When samples were irradiated by $\mathrm{THz}$ wave, the path that terahertz wave went through the gas gets longer. Therefore, the absorption coefficient showed a trend of decline. This study showed that growth temperature had a larger influence on crystal growth, especially inclusions, and it also illustrated that THz-TDS could accurately and rapidly detect the change of the inclusions inside crystals.

\section{Conclusions}

Fluid inclusion is one of the important parameters for studying the evolution process of mineral crystal. In this paper, $\mathrm{THz}-$ TDS was used to study sodium sulfate crystals with different growth temperatures. The results showed that terahertz absorption coefficients of sodium sulfate crystals exhibit the tendency of increasing first and then decrease with the increase of growth temperature, and the turning point was $80^{\circ} \mathrm{C}$, which was closely related with fluid inclusions inside sodium sulfate crystals. It also showed that terahertz had a unique advantage in studying crystal growth and geological diagenetic and metallogenic evolution, and it will thus likely see greater applications in this area.

\section{Acknowledgements}

This research was supported by the National Natural Science Foundation of China (Grant No. 61405259).

\section{References}

1. G. X. Chi, I. M. Chou, and H. Z. Lu, Acta Petro. Sin., 2003, 2, 201.

2. Z. Q. Xuan, Geol, Chem. Miner, 1997, 19, 203.

3. H. Z. Lu, H. R. Fan, and P. Ni, "Fluid Inclusions (in Chinese)", 2004, Science Press, Beijing.

4. J. J. Zambito and K. C. Benison, Geology, 2013, 41, 587.

5. E. L. Johnson and L. S. Hollister, J. Metamorph. Geol., 2010, 2, 239.

6. A. Fall, B. Tattitch, and R. J. Bodnar, Geochim. Cosmochim. Acta, 2011, 4, 951.

7. M. A. Pelch, M. S. Appold, P. Emsbo, and R. J. Bodnar, Econ. Geol., 2015, 3, 787.

8. J. Bourdet, R. C. Burruss, I. M. Chou, R. Kempton, K. Liu, and N. V. Hung, Geochim. Cosmochim. Acta, 2014, 142, 362.

9. N. Horiuchi and X. C. Zhang, Nat. Photonics, 2010, 4, 662.

10. M. C. Nuss and J. Orenstein, "Terahertz Time-domain Spectroscopy", 1998, Springer, Berlin, Heidelberg.

11. P. H. Siegel, IEEE. T. Microw. Theory, 2004, 52, 2438.

12. H. L. Zhan, S. X. Wu, R. M. Bao, L. N. Ge, and K. Zhao, Fuel, 2015, 143, 189.

13. C. Jiang, K. Zhao, L. J. Zhao, W. J. Jin, Y. P. Yang, and S. H. Chen, Energ. Fuel, 2014, 28, 483.

14. W. J. Jin, K. Zhao, C. Yang, C. H. Xu, H. Ni, and S. H. Chen, Appl. Geophys., 2013, 10, 506.

15. H. Zhao, K. Zhao, and R. M. Bao, J. Infrared Millim. Terahertz Waves, 2012, 33, 522.

16. R. M. Bao, S. X. Wu, K. Zhao, L. J. Zheng, and C. H. Xu, Sci. China-Phys. Mech. Astron., 2013, 56, 1603. 